\title{
BMJ Open Emergency department presentation and readmission after index psychiatric admission: a data linkage study
}

\author{
Xue Li, ${ }^{1}$ Preeyaporn Srasuebkul, ${ }^{1}$ Simone Reppermund, ${ }^{1,2}$ Julian Trollor ${ }^{1,2}$
}

\begin{abstract}
To cite: Li X, Srasuebkul P, Reppermund S, et al. Emergency department presentation and readmission after index psychiatric admission: a data linkage study. BMJ Open 2018;8:e018613. doi:10.1136/ bmjopen-2017-018613

- Prepublication history for this paper is available online. To view these files, please visit the journal online (http://dx.doi org/10.1136/bmjopen-2017018613).
\end{abstract}

Received 10 July 2017 Revised 7 January 2018 Accepted 11 January 2018

Check for updates

${ }^{1}$ Department of Developmental Disability Neuropsychiatry, School of Psychiatry, University of New South Wales, Sydney, New South Wales, Australia ${ }^{2}$ Centre for Healthy Brain Ageing, School of Psychiatry, University of New South Wales, Sydney, New South Wales, Australia

Correspondence to Professor Julian Trollor; J.Trollor@unsw.edu.au

\section{ABSTRACT}

Objective To use linked administrative datasets to assess factors associated with emergency department (ED) presentation and psychiatric readmission in three distinctive time intervals after the index psychiatric admission.

Design A retrospective data-linkage study.

Setting Cohort study using four linked government minimum datasets including acute hospital care from July 2005 to June 2012 in New South Wales, Australia. Participants People who were alive and aged $\geq 18$ years on 1 July 2005 and who had their index admission to a psychiatric ward from 1 July 2007 to 30 June 2010. Outcome measures ORs of factors associated with psychiatric admission and ED presentation were calculated for three intervals: 0-1 month, 2-5 months and 6-24 months after index separation.

Results Index admission was identified in 35056 individuals (51\% -males) with a median age of 42 years. A total of $12826(37 \%)$ individuals had at least one ED presentation in the 24 months after index admission. Of those, 3608 (28\%) presented within 0-1 month, 6350 (50\%) within 2-5 months and 10294 (80\%) within 6-24 months after index admission. A total of 14153 (40\%) individuals had at least one psychiatric readmission in the first 24 months. Of those, 6808 (48\%) were admitted within 0-1 month, 6433 (45\%) within 2-5 months and 7649 (54\%) within 6-24 months after index admission. Principal diagnoses and length of stay at index admission, sociodemographic factors, Charlson Comorbidity Index score, drug and alcohol comorbidity, intellectual disability and other inpatient service use were significantly associated with ED presentations and psychiatric readmissions, and these relationships varied somewhat over the intervals studied.

Conclusion Social determinants of service use, drug and alcohol intervention, addressing needs of individuals with intellectual disability and recovery-oriented wholeperson approaches at index admission are key areas for investment to improve trajectories after index admission.

\section{INTRODUCTION}

Mental illness is one of the leading cause of disability in developed countries. ${ }^{12}$ Building a mental health system that cohesively supports individuals with severe mental illness is a challenge for mental health services. Despite enhancements in community psychiatric

\section{Strengths and limitations of this study}

This study identifies factors associated with emergency department (ED) presentations and psychiatric readmissions following index admission after controlling for potential confounding factors in a large population-based dataset.

- This study represents the only study internationally that has examined ED presentation and psychiatric readmission at multiple time intervals after index separation.

- The major limitation of this study is the use of administrative data which lacks potentially important clinical information

supports, demand for acute services for people with mental ill health remains high, as evidenced by escalating use of emergency departments $(\mathrm{ED})^{3}$ and high demand for acute psychiatric inpatient services. ${ }^{4-6}$ Most acute psychiatric episodes of care are by repeat users. ${ }^{78}$ Thus, the development of a clear understanding of the drivers of ED use and psychiatric readmission for those with mental illness is of potential benefit to mental health consumers, service providers and health service administrators.

Administrative data can provide substantial insights into factors associated with mental health service use. Factors such as sociodemographic factors, specific psychiatric conditions, comorbidities and characteristics of previous hospital admissions all have significant impacts on mental health-related service use. ${ }^{9-12}$ For example, the 2010 Australian National Survey of Psychosis ${ }^{13}$ revealed that being younger, having high severity of psychotic symptoms and poor social functioning were associated with greater mental health service use. Another US study found among individuals with a mental health or substance abuse diagnosis psychiatric conditions such as schizophrenia and affective disorders not only increased the likelihood of psychiatric readmission, but also found to 
be predictors of ED presentations. ${ }^{12}$ Other strong predictors with a robust and reciprocal impact on both ED use and psychiatric service use are comorbid conditions including physical and psychiatric comorbidity, cognitive and psychiatric comorbidity, intellectual disability (ID) and drug and alcohol comorbid conditions. ${ }^{14} 15$ For example, a Canadian ${ }^{14}$ study found that individuals with ID and mental illness were more likely to have ED presentations and psychiatric admissions when comparing to individuals with ID only and mental illness only. Research relating to specific factors associated with acute mental health service use and ED use after index psychiatric admission has yet to be undertaken.

Rising demand for acute healthcare services and the substantial costs associated with repeat acute healthcare emphasise the importance of cohesive mental health supports and early intervention. ${ }^{16}$ Within the mental health context, the first psychotic episode is well recognised as a key opportunity for intervention with early engagement in recovery-oriented support resulting in demonstrated improvements in outcome ${ }^{17}$; however, this concept has broader relevance for a range of mental disorders. For many individuals, index admission represents a sentinel opportunity for mobilisation of first episode supports, yet little is known about service system trajectories after first admission. Understanding drivers of representation to acute psychiatric services will help to develop services appropriate to needs with their index admission, will enable potential strategies to improve service efficiency ${ }^{1819}$ and will potentially improve outcomes for affected individuals.

To date, emphasis has been placed on early readmission rates such as readmission within 28 days or 30 days as indicators of acute care service efficiency. ${ }^{18}$ However, predictors of readmission can be different at different time intervals following discharge.$^{20} \mathrm{~A}$ recent study by Kadam et $a l^{21}$ of acute healthcare service use and unplanned hospital admissions suggests that future research should include longer readmission intervals. Time intervals such as 6, 12 and 24 months after an admission have been used by various studies to gain a more comprehensive perspective on the service trajectory of a cohort. ${ }^{20}{ }^{22-24}$ It is likely that sociodemographic factors and physical and mental health comorbidities may interact to produce increasing complexity over time, with associated increases in the likelihood of representation to acute services. Thus, examination of the factors associated with acute mental health service use over several intervals, and for a substantial time period, is an important step in development of comprehensive understanding of the drivers of service use.

Population health administrative records in Australia provide an opportunity to examine acute health services use and their determinants. The current study aims to identify the factors associated with acute care service use following index psychiatric admission. Doing so will provide an opportunity to understand drivers of acute service use in this context and to better plan services and policy responses which underpin recovery from an initial mental health episode. To understand the dynamics of acute service use, it is important to examine the factors associated with ED presentation and psychiatric readmissions over multiple intervals in the 24 months after index separation. We hypothesised that sociodemographic factors, principal psychiatric diagnoses at index admission, length of stay at index admission, comorbidities and non-psychiatric admissions would have a significant association with ED presentations and psychiatric readmissions and that predictors of these may vary over time.

\section{METHODS}

\section{Datasets and record linkage}

Four linked datasets were used to define the cohort and/or exposure in this study. Deidentified linkage was performed by the New South Wales (NSW) Centre for Health Record Linkage based on a statistical linkage key (SLK581) ${ }^{25}$ In accordance with best practice privacy preserving protocols, the linked unit record data were provided to the researchers after removal of personal identifiers. The databases contained data collected from 1 July 2005 to 30 June 2012 in NSW. The databases used in this analysis were the following:

The Admitted Patient Data Collection (APDC) contains information on all admissions to public and private hospitals in NSW including psychiatric facilities. It also contains information on psychiatric, drug and alcohol and ID diagnoses. Diagnoses in this data collection were coded in the International Statistical Classification of Diseases and Related Health Problems, Tenth Revision, Australian Modification (ICD-10-AM). ${ }^{26}$

The Emergency Department Data Collection (EDDC) contains information on ED presentations at NSW public hospitals.

The Disability Services Minimum Dataset (DS MDS) is a state service data collection scheme that is collected by a disability administrator in each Australian jurisdiction. It contains information on ID diagnosis, which was used in conjunction with the APDC and EDDC to identify ID status.

The Registry of Birth Death and Marriage contain registration of death information, which was used to determine the period of exposure for this study.

\section{Study population}

The potential study population was people in the APDC with at least one recorded psychiatric occurring before 30June 2012 and who were alive at 1 July 2005. From this group, we excluded people aged $<18$ years on 1 July 2005 . Further, to determine those likely to be experiencing their 'index' or first-ever psychiatric admission in our observation period, we applied a 2-year look-back period and excluded from the final cohort those who were admitted to a psychiatric ward before 1 July 2007 . To ensure a 2-year minimum follow-up period, we also excluded those with first admission after 30 June 2010. 
Information regarding admission and separation from a psychiatric ward was obtained from the APDC record. Index admission was therefore a psychiatric admission occurring between 1 July 2007 and 30 June 2010, following a 2-year, admission-free look-back period. The index admission started at the date of the first admission to the psychiatric facility and ended when the index separation was noted.

Three different intervals were used in the study: (1) 0-1 month: this interval started at the date of the index separation to the 29th day after the index separation date; (2) 2-5 months: this interval started on the 30th day after the index separation to the 29th day of month 5; (3) 6-24 months: this interval started at the 30th day of the fifth month after the index separation date to the 29th day of month 23.

\section{Outcome measures}

There were two outcomes in this study; ED presentations and readmission to a psychiatric facility after the index separation. We considered patients who had any records of being admitted to a psychiatric facility after the index separation in each time interval as having a readmission to a psychiatric facility in the specific period. Similarly, patients who had any records of ED presentations after the index separation in each time interval were considered as having an ED presentation outcome. An individual could have more than one type of outcome and could have multiple admissions within one interval and across the time span.

\section{Statistical analysis}

Logistic regression was used to examine the factors associated with ED presentation and psychiatric admission for the three intervals -0 to 1 month, 2 to 5 months and 6 to 24 months after the index separation. Covariates included age, sex, the Index of Relative Socioeconomic Disadvantage (IRSD), ${ }^{27}$ remoteness area, principal psychiatric diagnoses at index admission, length of stay at index admission, Charlson Comorbidity Index score, ${ }^{28}$ ID status and drug and alcohol comorbidity.

The principal diagnosis was defined as the condition mainly responsible for a patient's episode of care in hospital. ${ }^{29}$ Principal psychiatric diagnoses at the index admission were identified using ICD-10-AM from the APDC dataset recorded at index admission. The codes from F00-F99 were grouped into seven categories: organic mental health disorder (F00-F09); drug and alcohol-related disorder (F10-F19); schizophrenia, schizotypal and delusion disorder (F20-F29); mood disorder (F30-F39); anxiety and stress-related disorder (F40-48); disorders of adult personality and behaviour (F60-F69). All other F codes were coded as other psychiatric disorders. Individuals who were given non-psychiatric codes were coded as not having a psychiatric disorder. The length of stay at index admission was calculated from the admission day to the separation day. Same-day admission was considered as 1 day.
Comorbidity scores in each interval were calculated using the modified Charlson Comorbidity Index score. ${ }^{28}{ }^{30}$ Comorbidities were identified from the APDC using ICD-10 codes. In addition to the Charlson Comorbidity Index, we examined two comorbidities, drug and alcohol and ID status, which were known to be highly associated with psychiatric readmissions or ED presentations. ${ }^{31} 32$

Drug and alcohol comorbidity coding was derived differently for the analyses of ED presentation and psychiatric readmission outcomes. For ED presentation outcomes, drug and alcohol comorbidity codes were derived from the APDC and were obtained by hospital episodes where drug and alcohol appeared in one of the diagnoses regardless of the admission type. However, for psychiatric readmission outcomes, to avoid conflation with the primary reason for psychiatric admission, we excluded drug and alcohol comorbidity diagnoses when these were the primary reason for admission to the psychiatric facility. Consistent with our previous approach, we identified ID with codes including childhood disintegrative and overactive disorders associated with mental retardation; intellectual development delay; mild through profound mental retardation; Down syndrome and other chromosomal anomalies associated with mental retardation; fragile $\mathrm{X}$ syndrome and congenital malformation syndromes due to known exogenous causes. ${ }^{33}$ The value of the ID flag was set to 1 throughout the study period for everyone who had any records with the relevant codes and set to 0 for everyone who did not have such a record. We also defined a binary variable representing any non-psychiatric hospital episodes for each individual using the APDC dataset. All other variables including age, sex, IRSD and remoteness were identified through the patient record from DS MDS, APDC and EDDC. Age was a time-dependent variable, it was recalculated at each time interval and was categorised into three groups: young adults (18-35 years); middleaged adults ( $36-55$ years); older adults ( $56+$ years).

All statistical analyses were completed with Stata V.14.0. ORs with $95 \%$ CI were reported, and the threshold for statistical significance was set at $\mathrm{P}<0.05$.

\section{RESULTS}

\section{Cohort characteristics}

There were 114095 individuals with at least one psychiatric admission from 1 July 2005 to 30 June 2012 and who were alive at 1 July 2005 . Of these, a total of 35056 individuals met inclusion criteria for the study. Of the 79039 excluded individuals, 13116 were excluded due to being under the age of 18 years on 1 July $2005 ; 65812$ were excluded for not having their first record admission between 1 July 2007 and 30 June 2010 and 111 were excluded for having an unknown area of residence and/or IRSD. For those meeting inclusion criteria, half of the population were male with the median and IQR of age at the beginning of the study period of 38 years (28-50 years) and at index admission, 42 years 


\section{Table 1 Cohort characteristics}

\begin{tabular}{lr}
\hline & \multicolumn{1}{l}{ Total } \\
\hline Number of people & $35056(100)$ \\
\hline Male & $17822(51)$ \\
\hline Median (IQR) age, years & \\
\hline At July 2005 & $38(28-50)$ \\
\hline At index admission & $42(32-54)$ \\
\hline $\begin{array}{l}\text { Median (IQR) length of stay at index } \\
\text { admission, days }\end{array}$ & $9(2-21)$ \\
\hline Remoteness of area & \\
\hline Major cities & $26468(76)$ \\
\hline Inner regional & $6778(19)$ \\
\hline $\begin{array}{l}\text { Outer regional/remote/very remote } \\
\text { Index of relative socioeconomic } \\
\text { disadvantage }\end{array}$ & $1810(5)$ \\
\hline 1-2 most disadvantaged & \\
\hline 3-4 & $5686(16)$ \\
\hline 5-6 & $5655(16)$ \\
\hline 7-8 & $8644(25)$ \\
\hline 9-10 least disadvantaged & $7332(21)$ \\
\hline Principal diagnoses at index admission & $7739(22)$ \\
\hline Mood disorder & $12707(36)$ \\
\hline Schizophrenia and delusion & $6998(20)$ \\
\hline $\begin{array}{l}\text { Disorder of adult personality and } \\
\text { behaviour }\end{array}$ & $1056(3)$ \\
\hline Drug and alcohol & $5070(14)$ \\
\hline $\begin{array}{l}\text { Anxiety and stress-related psychiatric } \\
\text { disorder }\end{array}$ & $7363(21)$ \\
\hline Organic psychiatric disorder & $667(2)$ \\
\hline Other psychiatric disorder & $740(2)$ \\
\hline Not psychiatric disorder & $455(1)$ \\
\hline Drug and alssion) & $6475(22)$ \\
\hline Intellectual disability & $13858(41)$ \\
\hline
\end{tabular}

$\mathrm{n}(\%)$ unless otherwise stated.

ED, emergency department.

Table 2 Percentiles of the number of emergency department presentations by intervals

\begin{tabular}{lccc}
\hline Percentile & 0-1 month & $\begin{array}{l}\text { 2-5 months } \\
\text { (4 months) }\end{array}$ & $\begin{array}{c}\text { 6-24 months } \\
\text { (18 months) }\end{array}$ \\
\hline 25th percentile & 1 & 1 & 1 \\
\hline 50th percentile & 1 & 1 & 2 \\
75th percentile & 2 & 3 & 4 \\
99th percentile & 7 & 13 & 27 \\
Maximum & 25 & 103 & 329 \\
\hline
\end{tabular}

(32-54 years). The majority of people in our cohort lived in major cities $(76 \%), 16 \%$ lived in the most disadvantaged area and 22\% lived in the least disadvantaged area. Mood disorders accounted for over a third of principal diagnoses for the index admission (36\%); followed by anxiety and stress-related psychiatric disorder $(21 \%)$, while disorders of adult personality and behaviour accounted for $3 \%$. One per cent of people in our cohort were coded as not having a psychiatric disorder. The median (IQR) length of stay at index admission was 9 days (2-21 days). Percentages of drug and alcohol use were $41 \%$ and $22 \%$ for the ED presentation outcome and psychiatric readmission, respectively. People with ID represented 3\% of the cohort (table 1).

\section{Factors associated with ED presentations and psychiatric readmissions after the index admission \\ ED presentation}

A total of $12826(37 \%)$ individuals had at least one ED presentation in the 24 months after index admission. Of those, 3608 (28\%) had ED presentations in the first month after the index admission with the median of 1 ED presentation per individual. Respectively, 6350 $(50 \%)$ and $10294(80 \%)$ individuals had ED presentations in the intervals of 2-5 months and 6-24 months after the index admission with medians of 1 and 2 ED presentations. Table 2 reports the percentiles of the number of ED presentation in each interval. The median time (IQR) to an ED presentation after the index admission was 107 (24-296) days.

Table 3 reports the ORs, CIs and $\mathrm{P}$ values of the factors associated with ED presentations after the index separation in the three intervals. Males were less likely than females to present to ED in the 2-5 months' and 6-24 months' intervals. Compared with young adults (18-35 years), individuals who were middle aged and older were both significantly less likely to present to an ED across all intervals. Area of residence also showed a consistent association with ED presentation. Compared with individuals who lived in major cities, individuals who lived in inner regional areas had increased likelihood of ED presentation after the index separation across all intervals. Individuals who lived in outer regional areas were more likely to have ED presentations in the first month after the index separation compared with those who lived in major cities. Area socioeconomic status had a considerable bearing on ED presentation such that those who lived in the least socioeconomic disadvantaged areas were less likely to present to an ED after the index separation than those who lived in the most disadvantaged areas across all intervals.

The principal diagnosis given at the index psychiatric admission had a significant association with ED presentations. Compared with mood disorders, disorders of adult personality and behaviour were consistently associated with elevated ED presentations across the three intervals. Longer length of stay at index admission reduced the likelihood of ED presentations across the study period. 


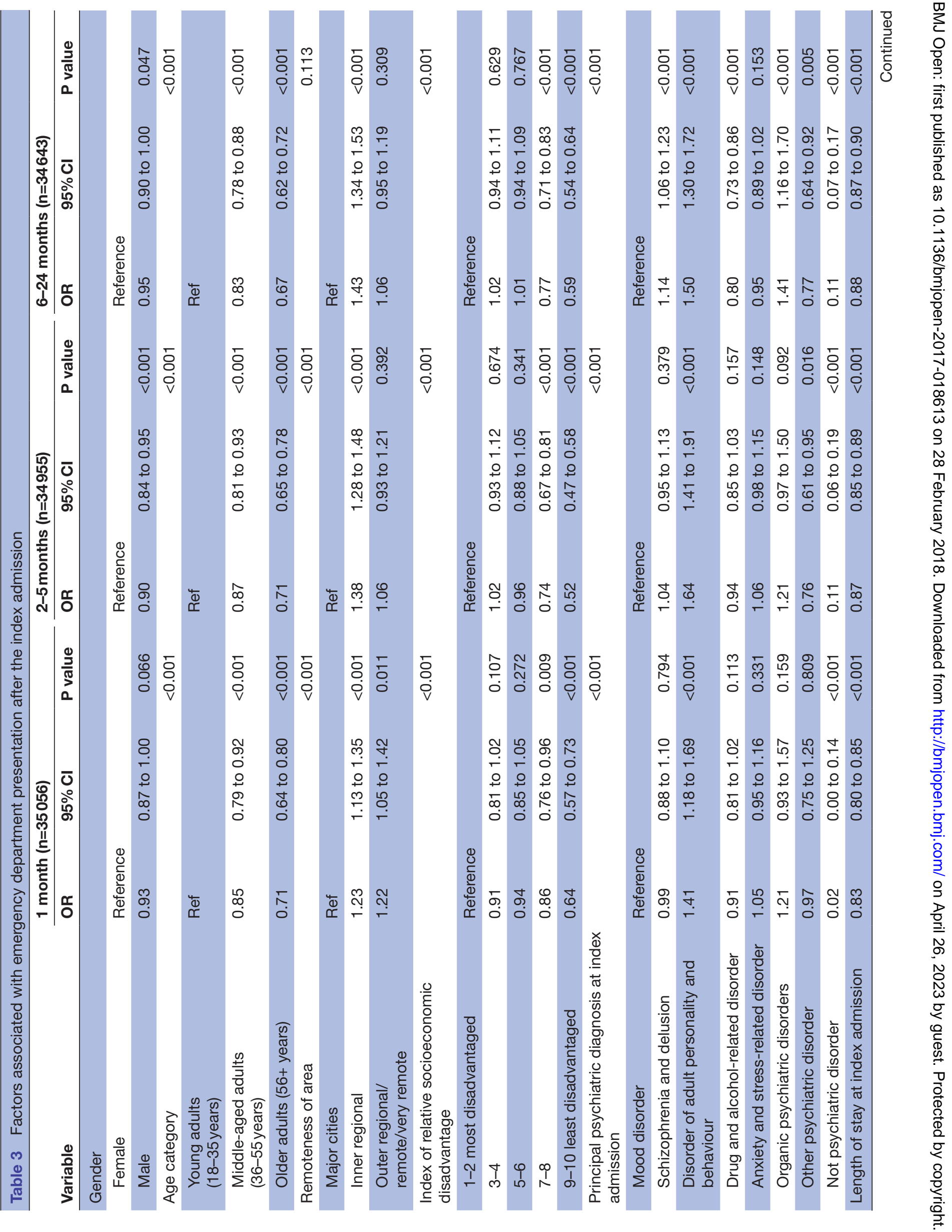




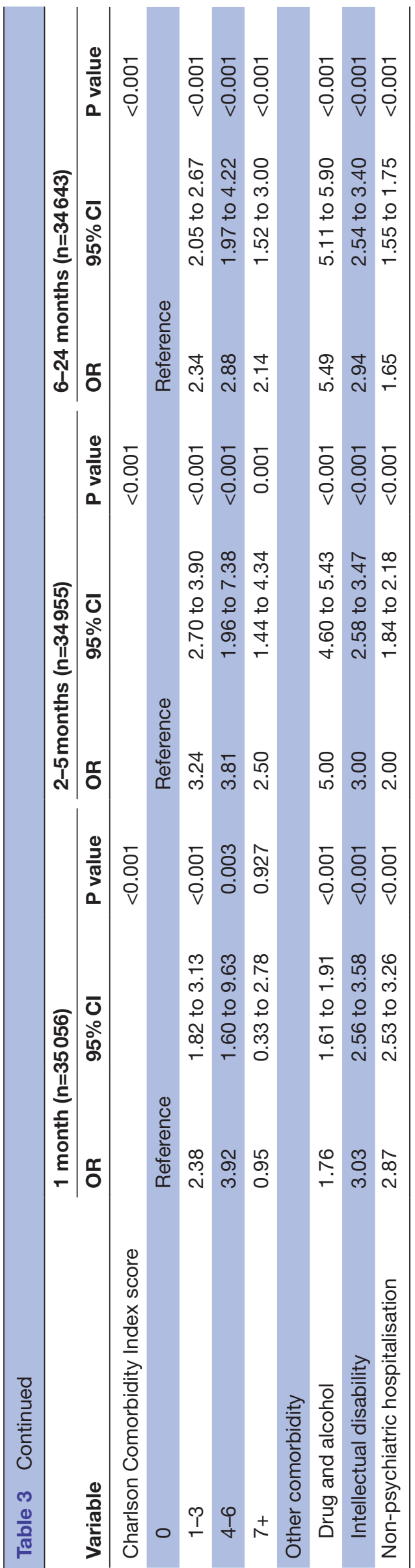

Table 4 Percentiles of the number of psychiatric readmission by intervals

\begin{tabular}{lccc}
\hline $\begin{array}{l}\text { Psychiatric } \\
\text { readmission }\end{array}$ & 0-1 month & $\begin{array}{l}\text { 2-5 months } \\
\text { (4 months) }\end{array}$ & $\begin{array}{l}\text { 6-24 months } \\
\text { (18 months) }\end{array}$ \\
\hline 25th percentile & 1 & 1 & 1 \\
50th percentile & 1 & 2 & 2 \\
75th percentile & 3 & 5 & 3 \\
99th percentile & 16 & 24 & 53 \\
Maximum & 23 & 94 & 157 \\
\hline
\end{tabular}

Comorbidity had an incremental association with ED presentation after the index separation. Comparing to individuals with no other physical illness (Charlson Comorbidity Index score of 0 ) recorded, individuals with a Charlson Comorbidity Index score of 1 to 3 were more likely to present to an ED across the three intervals. Having a Charlson Comorbidity Index score above 4 was associated with greater likelihood of ED presentation in the last two intervals. ID had a consistent and robust association with ED presentation across all intervals. The association between drug and alcohol comorbidity and ED presentation increased as time progressed.

Non-psychiatric hospitalisations were associated with ED presentations across all intervals; however, the strongest association was in the first month after the index admission and the ORs decreased with time.

\section{Psychiatric readmissions}

In the study period, 14153 (40\%) individuals had at least one psychiatric readmission in the 24 months after the index admission. Of those, 6808 (48\%) individuals had readmissions in the first month (median of 1 ) readmission. A total of $6433(45 \%)$ individuals had readmissions 2-5 months after the index admission (median of 2) and 7649 (54\%) had readmissions 6-24 months after the index admission (median of 2). Table 4 reports the percentiles of the number of readmissions in each interval. The median (IQR) time to a psychiatric readmission after the index admission was 36 (4-209) days.

Table 5 reports the ORs, CIs and $\mathrm{P}$ values of the factors associated with psychiatric readmissions. Males were consistently less likely to be readmitted to a psychiatric facility compared with females. Compared with young adults (18-35 years), older adults ( 56 years and above) and individuals aged between 35 and 55 were more likely to be readmitted in the first two intervals. Individuals who lived in the outer regional, remote and very remote areas were less likely to have a psychiatric readmission after the index separation than individuals who lived in major cities. Individuals who lived in the least disadvantaged area were more likely to be readmitted to a psychiatric facility than individuals who lived in the most disadvantaged area.

Principal psychiatric diagnosis at index admission was associated with psychiatric readmission. Compared with mood disorders, both schizophrenia and delusion 


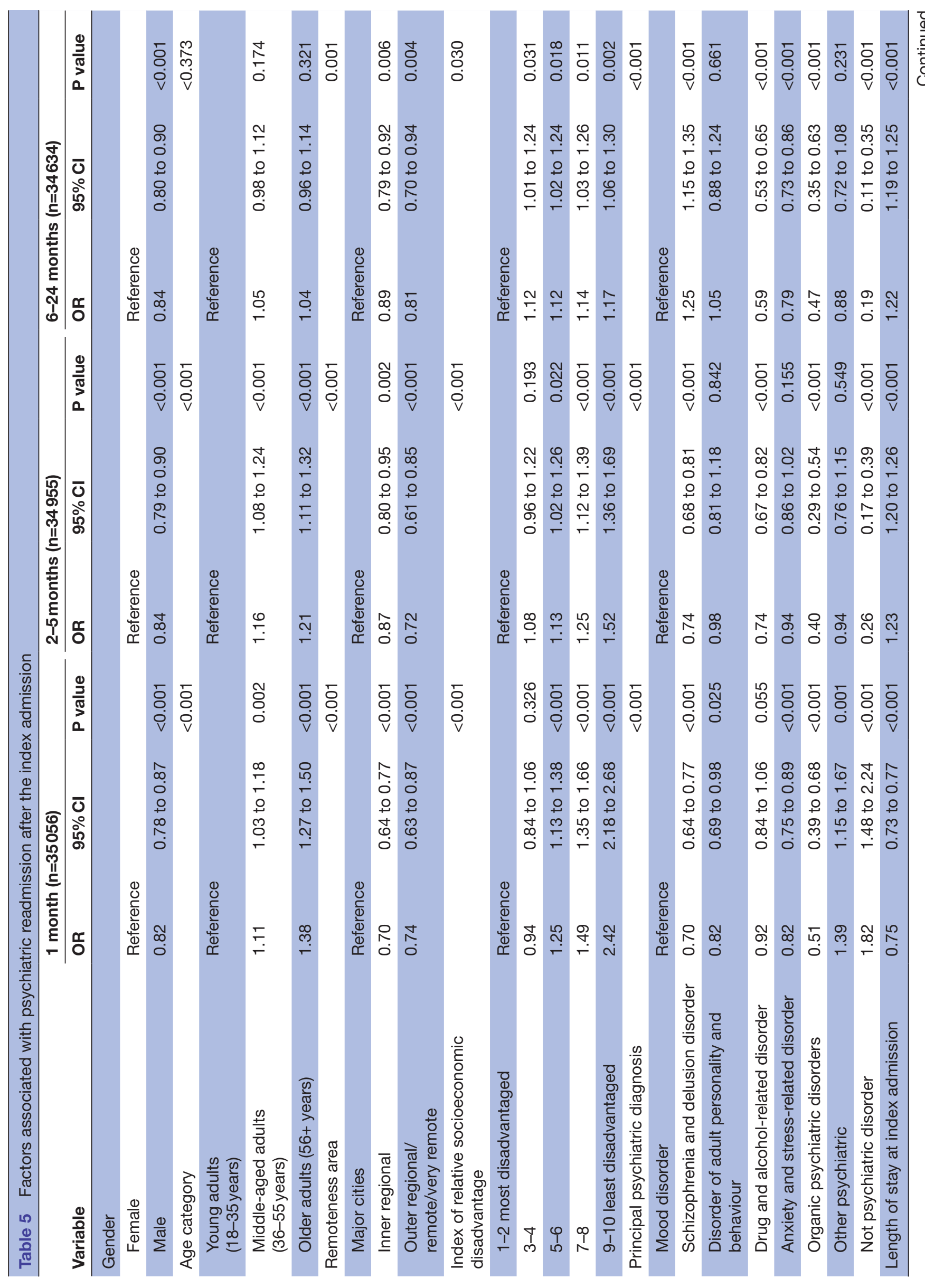




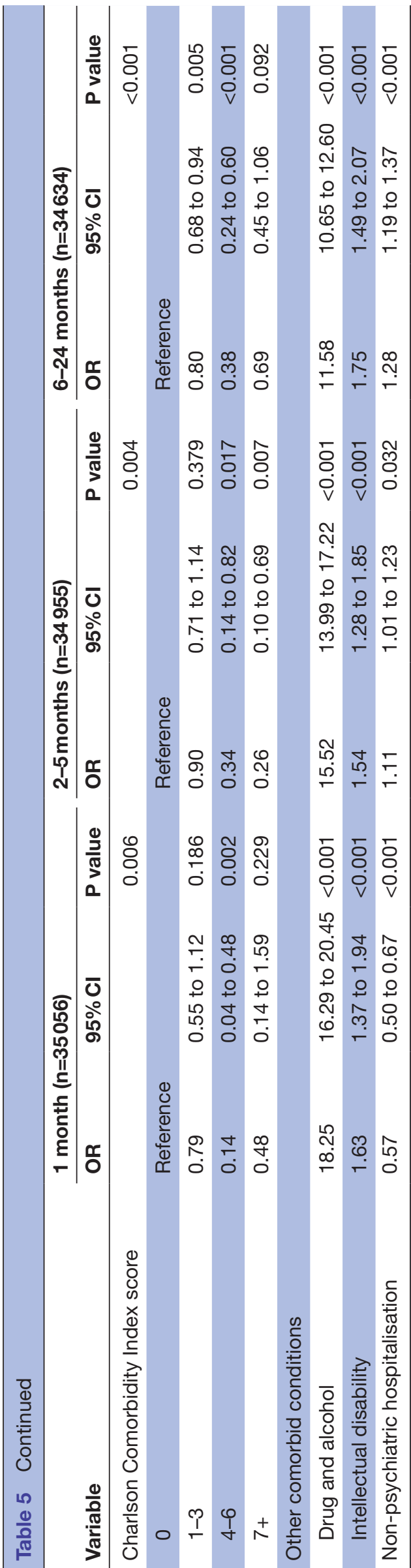

disorder and organic psychiatric disorder had a persistent association with psychiatric readmission. Schizophrenia and delusion disorder was associated with lower likelihood of readmission within the first 6 months but was associated with higher likelihood in the last interval. Organic psychiatric disorder diagnosis was associated with lower likelihood psychiatric readmissions across the study period. A principal diagnosis in the drug and alcohol category was associated with greater likelihood psychiatric readmission in the last two intervals. Anxiety and stress-related psychiatric disorder were significantly associated with reduced likelihood of readmission in the first and last interval. Having a 'Not psychiatric diagnosis' at index admission increased the likelihood of readmission in the first month and decreased the likelihood of readmission in the last two intervals. Greater length of stay at index admission was associated with lower likelihood of readmissions in the first interval but with increased likelihood of readmission in the last two intervals.

Higher Charlson Comorbidity Index scores were generally associated with a lower likelihood of psychiatric readmissions. ID was consistently associated with higher rates of psychiatric readmissions across all intervals. There were strong associations between and psychiatric readmissions and drug and alcohol comorbidity. Drug and alcohol comorbidity significantly increased the likelihood of readmission across all intervals.

Non-psychiatric hospitalisations were associated with decreased likelihood of psychiatric readmissions in the first interval and increased likelihood of readmission in the last two intervals.

\section{DISCUSSION}

Sociodemographic factors, principal psychiatric diagnoses at index admission, length of stay at index admission, comorbidities and non-psychiatric inpatient admissions were all significantly associated with ED presentation and psychiatric readmission. While some factors had a robust and consistent association across all time intervals, each interval revealed a distinctive pattern for some of these associations.

Similar to previous studies demonstrating a relationship between sociodemographic and mental health service use ${ }^{34}{ }^{35}$ this study found that age and sex had a significant association with ED presentations and psychiatric readmissions after the index admission. Extending previously documented associations between sex and mental health service use ${ }^{13}$ being male was associated with lower likelihood of psychiatric readmissions in our study. Consistent with previous population data,${ }^{12}$ being younger increased the likelihood of ED presentation but was associated with a reduced likelihood of readmission in the short to medium term.

While low socioeconomic status and remoteness of the living area were associated with more ED presentations, they were associated with lower likelihood of psychiatric readmissions. Our findings are consistent 
with previous studies which found that individuals with higher education and income use more mental health services $^{36}{ }^{37}$ whereas individuals with lower socioeconomic status tend to use more crisis-driven services such as ED. ${ }^{38}{ }^{39}$ EDs are widely distributed and freely available through a universal healthcare system in Australia. Attendance at ED is patient or carer initiated and is available regardless of socioeconomic status. In contrast, inpatient psychiatric care is available in larger centres only and is provided by both public and private providers, the latter of which are accessed only by those able to afford private health insurance and/or copayment for services. ${ }^{40}$ Further, the decision to admit to inpatient psychiatric care is typically made on the basis of a comprehensive assessment of clinical needs. Together, these factors may explain the variable relationships of ED representation and readmissions with socioeconomic status and remoteness of living area.

Principal psychiatric diagnosis had a persistent impact on the service trajectory of an individual. For example, the association between personality and behavioural disorder and ED presentation after index separation may relate to symptoms associated with self-harm which is a well-recognised reason for presentation to ED. ${ }^{41}$ However, reflecting the emphasis on enduring community-based supports in its management, personality and behavioural disorder was not associated with readmission. Compared with mood disorder diagnoses, schizophrenia and delusion disorder were associated with lower likelihood of readmissions in the first 6 months after the index admission. However, previous findings showed that schizophrenia as a principal diagnosis was highly correlated to ED presentation and psychiatric readmission within 30 days after index admission. ${ }^{12}$ This may be explained by the clustered code used in this study which combined the most common psychiatric disorders such as depression and bipolar disorder into one categorymood disorder-and it represents $36 \%$ of the cohort.

The association between length of stay at index admission and ED presentations differed from that observed with readmission and may have related to the interaction of initial severity/complexity of presentation (determining index admission length) and time-dependent factors such as subsequent clinical pathways. For individuals experiencing first psychiatric admission, subsequent allocation of community supports may be most cohesive for those with higher levels of complexity, for which length of index admission may be a proxy. This could have mitigated representation to ED and early rates of readmission. With time, it is possible that community supports become less cohesive over time, and indeed a weakening of the relationship between length of index admission and representation to ED was noted over time. While the same mitigation was initially apparent in the 1-month readmission data, this appeared to be swamped in subsequent time periods by other factors. Although this is harder to explain, it is possible that those individuals with greater complexity may have subsequently been more likely to present directly to psychiatric inpatient facilities.

Drug and alcohol-related disorders as principal diagnoses were associated with lower likelihood of psychiatric readmissions, and yet as a comorbid condition drug and alcohol-related diagnoses showed the highest association of all covariates with psychiatric readmission and ED presentation across the time intervals. Only $14 \%$ of the cohort had a principal drug and alcohol-related diagnosis, whereas $41 \%$ of the cohort had a drug and alcohol comorbidity in the ED data, suggesting that these two labels pick up different presentations in different contexts and with different clinical supports. The two highly correlated diagnoses of drug and alcohol use and mental ill health are often referred to as dual diagnosis in mental healthcare. ${ }^{42}$ It is understood that drug and alcohol comorbidity can lead to reductions of compliance with psychiatric treatment, and as a result dual diagnosis is often managed in inpatient mental health services. ${ }^{32}$ The strength of the association between drug and alcohol comorbidity and ED presentation and psychiatric readmission suggests that drug and alcohol intervention should occur early and should be a sustained focus in healthcare. Drug and alcohol comorbidity also had a stronger association with psychiatric readmission than ED presentation; such a difference may be partially explained by the proportion of individuals with complex needs being admitted to a psychiatric facility bypassing ED. However, more research is needed to investigate the factors attributed to this distinctive service-use pattern.

Previous studies ${ }^{15}$ reported a high prevalence of physical comorbidity among individuals with mental illness, and we found that the Charlson Comorbidity Index score had an opposite impact on ED presentation and psychiatric readmission. The Charlson Comorbidity Index score is often used to predict mortality rate within a year. ${ }^{43}$ Individuals with more severe physical comorbidities were understandably more likely to present to ED and less likely to be readmitted to a psychiatric facility. The current study was unable to investigate whether physical health comorbidities were related to the index admission or the onset of a psychiatric illness. The findings do however suggest that an emphasis on tailored and holistic healthcare is needed within both mental health services and primary healthcare settings.

The presence of ID was persistently and strongly associated with ED presentation and psychiatric readmission across the study period which is consistent with previous research. ${ }^{31}$ As reported elsewhere, ${ }^{44}{ }^{45}$ the mental health system in Australia is not yet equipped to provide comprehensive mental health supports for individuals with ID. Consistent with a previous study, ${ }^{14}$ the current study suggests that ID adds to complex support needs which have a direct bearing on ED and inpatient mental health service use, above and beyond that due to the mental illness alone. Unlike many physical conditions which an individual can acquire at any point in time, ID is a permanent disability that is often identified at an early stage in 
life. Our findings reaffirm that equipping mental health services to meet the mental health needs of people with ID is useful and may assist in buffering the impact of this disability on service use.

A commonly agreed definition of 'frequent users' is those with three or more visits per year for ED presentation. ${ }^{46} \mathrm{~A}$ quarter of the cohort had three or more ED presentations even within a short 2-5 months' period after index admission. Past studies have found that frequent ED users tend to have complex healthcare needs and are frequent users of primary and acute health services. ${ }^{47} 48$ The current study also found that non-psychiatric admissions increased the likelihood of ED presentations and psychiatric readmission. These findings suggest that strong relationships exist between each component of acute healthcare services and are in keeping with past research ${ }^{49}$ in which a small proportion of acute service users consumed intensive resources and were not optimally managed within the context of acute healthcare setting. Further research is needed to explore the characteristics of frequent service users in this cohort.

The change of direction of the association of principal diagnoses such as schizophrenia and delusion disorder and psychiatric readmission at different time intervals suggest that the service trajectories of individuals with different psychiatric disorders and symptoms can vary and that the 30-day readmission predictors may not capture such change. Further research should seek to explore in more detail the drivers and dynamics of fluctuations in service use over time.

\section{Strengths and limitations}

To our knowledge, this is the only cohort study internationally that has examined these associations at multiple time intervals. The current data-linkage study represents a large cohort and provides a comprehensive overview of factors associated with psychiatric readmissions and ED presentations. Our method of identifying index admissions may have inadvertently captured individuals who had admissions prior to July 2005. However, given that $60 \%$ of mental health service users in Australia had a mental health disorder lasting 12 months $^{8}$ and a previous study ${ }^{50}$ found that $66 \%$ of mental health users readmitted to a psychiatric facility within a year, it is unlikely that this has affected the results.

A limitation of the current study is the use of data collected for administrative rather than clinical purposes; therefore, we lack potentially important clinical information. We were unable to examine the severity of symptoms when admitted to the hospital and its association with readmissions and ED presentation. As APDC data are collected on separation from the hospital, we were also unable to identify the very small percentage of individuals who had an index admission during the study period and yet remained in the facility throughout the study period.

ID had a robust and persistent impact on both ED presentation and psychiatric readmission; however, due to the limitation of the research scope of this study, we did not further examine subgroups of people with ID. The results of the current study are a strong indicator of the unmet needs of the ID population. Further research that examines subpopulations such as individuals with autism, Down syndrome and Fetal Alcohol Syndrome (FAS) within the ID population is needed to understand their needs. We also acknowledge that a small proportion of individuals with borderline and mild ID may not be identified in the ID cohort if they did not receive disability services previously.

\section{CONCLUSIONS}

We propose the following recommendations to improve service integration: a stronger public health approach to address the impact of social determinants on service use, early intervention programmes for dual diagnosis of mental illness and drug and alcohol comorbidity, an urgent response to address the unmet needs of individuals with ID and mental illness and a more holistic care approach to address comorbidity in the inpatient setting. In addition, more research is needed to understand the service trajectories of individuals with different psychiatric conditions beyond the commonly used 30-day interval.

Acknowledgements We would like to acknowledge all investigators and partner organisations contributing to the project: Chief investigators: Eric Emerson, Rhoshel Lenroot, Karen Fisher, Kimberlie Dean, Leanne Dowse. Associate investigators: Eileen Baldry, Tony Florio, Grant Sara, Phillip Snoyman, Les White. Project staff and students: Angela Dew, Erin Whittle, , Bronwyn Newman, Theresa Heintze. Partner organisations: agency for Clinical Innovation—Intellectual Disability Network; NSW Department of Family and Community Services_-Ageing, Disability and Home Care; NSW Department of Education; NSW Department of Justice-Corrective Services NSW; NSW Ministry of Health-Justice Health and Forensic Mental Health Network, Mental Health Commission of NSW; NSW Ministry of Health-Mental Health and Drug and Alcohol Office; NSW Ministry of Health-InforMH, Mental Health Review Tribunal, National and NSW Council for Intellectual Disability; National Disability Services; NSW Office of the Public Guardian; NSW Ombudsman.

Contributors All authors participated in the conceptual design of the study. XL wrote the first draft of the manuscript. PS performed the analyses. All authors critically revised the manuscript and contributed to interpretation of the data. All authors read and approved the final version of the manuscript.

Funding This study was funded by a National Health and Medical Research Council Australia funded Partnerships for Better Health grant (ID: APP1056128; Title: Improving the Mental Health Outcomes of People with an Intellectual Disability).

Competing interests None declared.

Patient consent Not required.

Ethics approval Ethics approval was obtained from the NSW Population and Health Services Research Ethics Committee (AU RED Study Reference Number: HREC/13/CIPHS/7; CINSW Reference Number: 2013/02/446), and access to the data sets was granted by relevant data custodians.

Provenance and peer review Not commissioned; externally peer reviewed.

Data sharing statement Direct access to the data and analytical files is not permitted without the expressed permission of the approving human research ethics committees and data custodians. Researchers interested in collaboration should contact the corresponding author with their expression of interest.

Open Access This is an Open Access article distributed in accordance with the Creative Commons Attribution Non Commercial (CC BY-NC 4.0) license, which permits others to distribute, remix, adapt, build upon this work non-commercially, and license their derivative works on different terms, provided the original work is properly cited and the use is non-commercial. See: http://creativecommons.org/ licenses/by-nc/4.0/

(C) Article author(s) (or their employer(s) unless otherwise stated in the text of the article) 2018. All rights reserved. No commercial use is permitted unless otherwise expressly granted. 


\section{REFERENCES}

1. Jacobi F, Wittchen $\mathrm{H}-\mathrm{U}$, Holting $\mathrm{C}$, et al. Prevalence, co-morbidity and correlates of mental disorders in the general population: results from the German Health Interview and Examination Survey (GHS). Psychol Med 2004;34:597-611.

2. Whiteford HA, Degenhardt L, Rehm J, et al. Global burden of disease attributable to mental and substance use disorders: findings from the Global Burden of Disease Study 2010. Lancet 2013;382:1575-86.

3. Kalucy R, Thomas L, King D. Changing demand for mental health services in the emergency department of a public hospital. Aust N Z J Psychiatry 2005;39:74-80.

4. Whiteford HA. Australia's national mental health policy. Hosp Community Psychiatry 1993;44:963.

5. Whiteford HA, Buckingham WJ. Ten years of mental health service reform in Australia: are we getting it right? Med J Aust 2005;182:396-400.

6. Allison S, Bastiampillai T. Mental health services reach the tipping point in Australian acute hospitals: the OECD warns about Australia's low psychiatric bed numbers. Medical Journal of Australia 2015;203:432-4.

7. Jaramillo-Gonzalez LE, Sanchez-Pedraza R, Herazo MI. The frequency of rehospitalization and associated factors in Colombian psychiatric patients: a cohort study. BMC Psychiatry 2014;14:161.

8. Slade TJA, Teesson M, Whiteford H, et al. The Mental Health of Australians 2. In: DoHa A, ed. Report on the 2007 National Survey of Mental Health and Wellbeing. Canberra, 2009.

9. Lorine K, Goenjian H, Kim S, et al. Risk factors associated with psychiatric readmission. J Nerv Ment Dis 2015;203:425-30.

10. Régis EMB, oão AM, Isabela Panzeri $C$, et al. Short admission in an emergency psychiatry unit can prevent prolonged lengths of stay in a psychiatric institution Internação breve em unidade de emergência psiquiátrica pode prevenir permanência prolongada em instituições psiquiátricas. Revista Brasileira de Psiquiatria 2010;32:145-51.

11. Loch AA. Stigma and higher rates of psychiatric re-hospitalization: São Paulo public mental health system. Rev Bras Psiquiatr 2012;34:185-92.

12. Smith MW, Stocks C, Santora PB. Hospital readmission rates and emergency department visits for mental health and substance abuse conditions. Community Ment Health J 2015;51:190-7.

13. Raudino A, Carr VJ, Bush R, et al. Patterns of service utilisation in psychosis: findings of the 2010 Australian national survey of psychosis. Aust N Z J Psychiatry 2014;48:341-51.

14. Lunsky $Y$, Lin E, Balogh R, et al. Emergency department visits and use of outpatient physician services by adults with developmental disability and psychiatric disorder. Can J Psychiatry 2012;57:601-7.

15. Parslow RA, Jorm AF. Who uses mental health services in Australia? An analysis of data from the National Survey of Mental Health and Wellbeing. Aust N Z J Psychiatry 2000;34:997-1008.

16. Billings J, Blunt I, Steventon A, et al. Development of a predictive model to identify inpatients at risk of re-admission within 30 days of discharge (PARR-30). BMJ Open 2012;2:e001667.

17. McGorry P. Early intervention in psychosis. Aust N Z J Psych 2006;40:A109-A09.

18. Shadmi E, Flaks-Manov N, Hoshen M, et al. Predicting 30-day readmissions with preadmission electronic health record data. Med Care 2015;53:283-9.

19. van Walraven C, Dhalla IA, Bell C, et al. Derivation and validation of an index to predict early death or unplanned readmission after discharge from hospital to the community. CMAJ 2010;182:551-7.

20. Lin $\mathrm{CH}$, Chen WL, Lin CM, et al. Predictors of psychiatric readmissions in the short- and long-term: a population-based study in Taiwan. Clinics 2010;65:481-9.

21. Kadam UT, Lawson CA, Moody DK, et al. Consumer segmentation and time interval between types of hospital admission: a clinical linkage database study. J Public Health 2017;9:1-9.

22. Librero J, Peiró S, Ordiñana R. Chronic comorbidity and outcomes of hospital care: length of stay, mortality, and readmission at 30 and 365 days. J Clin Epidemiol 1999;52:171-9.

23. Tsao Cl, Chou NK, Chi NH, et al. Unplanned readmission within 1 year after heart transplantation in Taiwan. Transplant Proc 2010;42:946-7.

24. Ono T, Tamai A, Takeuchi D, et al. Factors related to readmission to a ward for dementia patients: sex differences. Psychiatry Clin Neurosci 2011;65:490-8.

25. The Centre for Health Record Linkage (CHeReL). How record linkage works. 2016. http://www.cherel.org.au/how-record-linkage-works (accessed 26 Oct 2017).
26. World Health Organization. The ICD-10 Classification of Mental and Behavioural Disorders: clinical descriptions and diagnostic guidelines. Geneva: World Health Organization, 1992.

27. Australian Bureau of Statistics. Socio-Economic Index for Areas. Canberra, Australia: Australian Bureau of Statistics, 2013.

28. Charlson ME, Pompei P, Ales KL, et al. A new method of classifying prognostic comorbidity in longitudinal studies: development and validation. J Chronic Dis 1987;40:373-83.

29. Australian Institute of Health and Welfare. Principal diagnosis data cubes. 2017. https://www.aihw.gov.au/reports/hospitals/principaldiagnosis-data-cubes/contents/data-cubes (accessed Oct 2017).

30. Quan H, Sundararajan V, Halfon P, et al. Coding algorithms for defining comorbidities in ICD-9-CM and ICD-10 administrative data. Med Care 2005;43:1130-9.

31. Lunsky Y, Balogh R, Cairney J. Predictors of emergency department visits by persons with intellectual disability experiencing a psychiatric crisis. Psychiatr Serv 2012;63:287-90.

32. Regier DA, Farmer ME, Rae DS, et al. Comorbidity of mental disorders with alcohol and other drug abuse. Results from the Epidemiologic Catchment Area (ECA) Study. JAMA 1990;264:2511.

33. Reppermund S, Srasuebkul P, Heintze T, et al. Cohort profile: a data linkage cohort to examine health service profiles of people with intellectual disability in New South Wales, Australia. BMJ Open 2017;7:e015627.

34. Turrell G, Oldenburg BF, Harris E, et al. Utilisation of general practitioner services by socio-economic disadvantage and geographic remoteness. Aust N Z J Public Health 2004;28:152-8.

35. Narrow WE, Regier DA, Norquist G, et al. Mental health service use by Americans with severe mental illnesses. Soc Psychiatry Psychiatr Epidemiol 2000;35:147-55.

36. Alegría M, Bijl RV, Lin E, et al. Income differences in persons seeking outpatient treatment for mental disorders: a comparison of the United States with Ontario and The Netherlands. Arch Gen Psychiatry 2000;57:383-91.

37. Vasiliadis HM, Lesage A, Adair C, et al. Do Canada and the United States differ in prevalence of depression and utilization of services? Psychiatr Serv 2007;58:63-71.

38. Australian Institute of Health and Welfare. Australian hospital statistics 2007-08. Canberra: AlHW, 2009. (accessed 12 Jan 2017).

39. Hadley J. Insurance coverage, medical care use, and short-term health changes following an unintentional injury or the onset of a chronic condition. JAMA 2007;297:1073-84.

40. Wilks CM, Oakley Browne M, Jenner BL. Attracting psychiatrists to a rural area: 10 years on. Rural Remote Health 2008;8:824.

41. Haq SU, Subramanyam D, Agius M. Assessment of self harm in an accident and emergency service: the development of a proforma to assess suicide intent and mental state in those presenting to the emergency department with self harm. Psychiatr Danub 2010;22(Suppl 1):S26-32.

42. Donald M, Dower J, Kavanagh D. Integrated versus non-integrated management and care for clients with co-occurring mental health and substance use disorders: a qualitative systematic review of randomised controlled trials. Soc Sci Med 2005;60:1371-83.

43. Quan H, Li B, Couris CM, et al. Updating and validating the Charlson comorbidity index and score for risk adjustment in hospital discharge abstracts using data from 6 countries. Am J Epidemiol 2011;173:676-82.

44. Howlett S, Florio $\mathrm{T}, \mathrm{Xu} \mathrm{H}$, et al. Ambulatory mental health data demonstrates the high needs of people with an intellectual disability: results from the New South Wales intellectual disability and mental health data linkage project. Aust N Z J Psychiatry 2015;49:137-44.

45. Chaplin R. Mental health services for people with intellectual disabilities. Curr Opin Psychiatry 2011;24:372-6.

46. Grover CA, Close RJ. Frequent users of the emergency department: risky business. West J Emerg Med 2009;10:193.

47. Soril LJ, Leggett LE, Lorenzetti DL, et al. Reducing frequent visits to the emergency department: a systematic review of interventions. PLoS One 2015;10.

48. Hansagi $\mathrm{H}$, Olsson M, Sjöberg S, et al. Frequent use of the hospital emergency department is indicative of high use of other health care services. Ann Emerg Med 2001;37:561-7.

49. Keene J. Clients with complex needs: interprofessional practice. Oxford: Oxford : Blackwell Science, 2001.

50. Wheeler A, Moyle S, Jansen C, et al. Five-year follow-up of an acute psychiatric admission cohort in Auckland, New Zealand. $N$ Z Med J 2011;124:30-8 